\title{
Maritime Anomaly Detection based on Mean-Reverting Stochastic Processes Applied to a Real-World Scenario
}

\author{
Enrica d'Afflisio*, Paolo Braca*, Leonardo M. Millefiori* and Peter Willett ${ }^{\dagger}$ \\ * NATO STO Centre for Maritime Research and Experimentation, La Spezia, Italy \\ $\dagger$ Department of Electrical and Computer Engineering, University of Connecticut, Storrs, USA
}

\begin{abstract}
A novel anomaly detection procedure is presented, based on the Ornstein-Uhlenbeck (OU) mean-reverting stochastic process. The considered anomaly is a vessel that deviates from a planned route, changing its nominal velocity. In order to hide this behavior, the vessel switches off its Automatic Identification System (AIS) device for a certain time, and then tries to revert to the previous nominal velocity. The decision that has to be taken is either declaring that a deviation happened or not, relying only upon two consecutive AIS contacts. A proper statistical hypothesis testing procedure that builds on the changes in the OU process long-term velocity parameter of the vessel is the core of the proposed approach and enables for the solution of the anomaly detection problem.
\end{abstract}

Index Terms-Ornstein-Uhlenbeck process, maritime anomaly detection, statistical hypothesis test, target tracking, automatic identification system, maritime security, real-world data.

\section{INTRODUCTION}

Ships involved in stealth activities [1], in which the perpetrators aim to remain hidden and undetected by lawenforcement bodies throughout the whole duration of the activity, tend to follow set patterns depending on the illicit activity in which they are engaged: deviation from standard routes, unexpected AIS activity, unexpected port arrival, close approach, and zone entry [2]. Such set patterns are associated to an anomalous behavior since the analysis of real-world AIS data shows that a significant portion of the vessels in open seas maneuver very seldom, tending to maintain a nearly constant speed.

Therefore, considering coverage gaps (e.g., counterfeit AIS reports, AIS device shutdown, limited radar coverage, etc.) an anomaly detection strategy turns out to be essential.

A real world example of anomalous behavior is provided in Fig. 1, where the about five-month track of a cargo vessel is shown. The vessel navigates with a nominal speed of about $5 \mathrm{~m} / \mathrm{s}$ in the waters of the Pacific Ocean [3]. Nearby the Galápagos Exclusive Economic Zone (EEZ), the vessel shuts the engines down and starts drifting, with an apparent deviation from its route. The reason of this deviation of the vessel is to rendezvous with four tuna longliners at about 1700 miles away from the Galápagos. Each fishing vessel spends about 12 hours moving along the cargo vessel at a distance of about $30 \mathrm{~m}$, which indicates the boats were likely tied
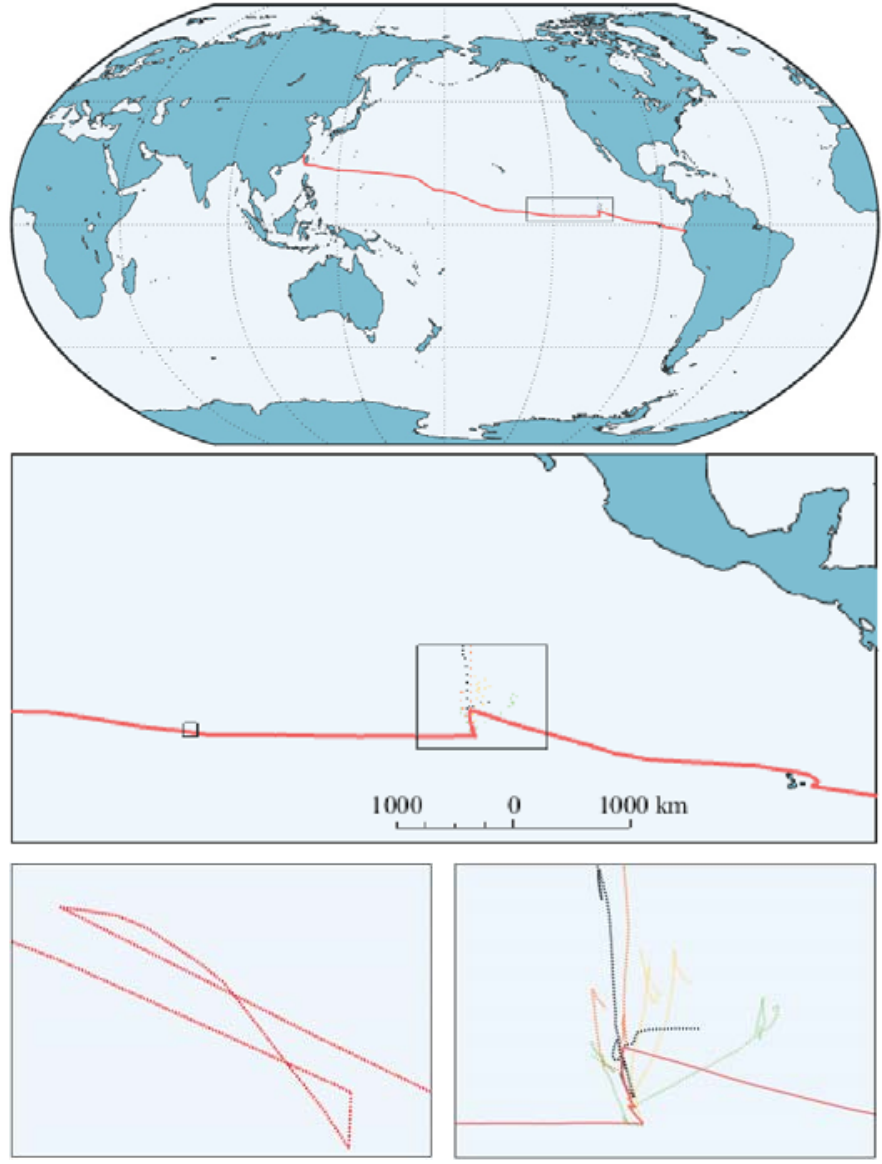

Fig. 1. The track of the cargo vessel (red) suspected of several rendezvous with four fishing vessels (dotted tracks).

up. This behavior suggests a substantial transfer of cargo was possible [3].

In this work, we consider the anomaly detection problem where a certain vessel switches off its AIS transponder for a certain amount of time, in order to hide its deviation from a planned route, which is characterized by a nominal velocity. The vessel would then try to revert back to the planned route and to the original nominal velocity. The decision to take is whether a deviation happened or not, relying only upon 


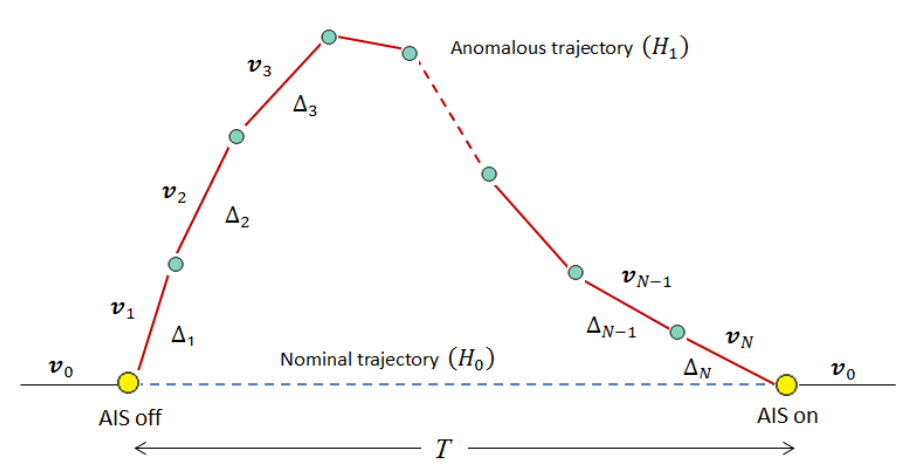

Fig. 2. Sequence of long-run mean velocities and time intervals characterizing the $N$-section path under hypothesis $H_{1}$.

two consecutive AIS contacts, i.e. the last contact before the AIS device shutdown and the first one after the AIS device reactivation. The extension to the case of other contacts available from sensors other than AIS is studied in [4]. The proposed anomaly detection strategy will be tested against a trajectory of a real transshipment [3] incident, based on a hypothesis testing procedure that builds on the changes of the Ornstein-Uhlenbeck (OU) process long-run mean velocity parameter.

The OU process has been shown to be better suited to model the behavior of a significant portion of real-world vessel trajectories than respect to conventional models [5][11]. In this framework, the use of the OU model turns out to be a valuable tool when vessel information is not available, providing a good estimation of a ship's position and velocity, even after several hours.

The rest of the paper is organized as follows. The problem is formulated in Section II, while Section III is devoted to the development of the detection strategy, and the analysis of the real scenario is reported in Section IV. Finally, conclusive remarks are provided in Section V.

\section{PROBLEM FORMULATION}

Let us consider a vessel of interest, represented by a point in a two-dimensional space, which is following its planned route. The position and velocity of the vessel are expressed in Cartesian coordinates, resulting from the projection of the geographic coordinates reported by the on-board AIS transponder. The OU stochastic process is used to represent the velocity of the vessel, with a long-run mean velocity $\boldsymbol{v}_{0}$ that represents the nominal velocity of the ship [5]. In other words, the velocity of the vessel is a modified Wiener process so that there is a tendency of the process to move back towards the long-run mean value, with a greater attraction when the process is further away from it. The velocity $\boldsymbol{v}_{0}$ is therefore a deterministic parameter of the OU stochastic process [5].

Let us now suppose that AIS data is unavailable for a time $T$ after a given instant, due to a lack of communications from the ship (because of limited sensor coverage, interference, etc.) or an intentional shutdown of the AIS transponder. In this context, two hypotheses can be envisioned: the first one, denoted by $H_{0}$, that the vessel navigates according to the nominal condition (along the planned trajectory with a longrun mean velocity $\boldsymbol{v}_{0}$ ); and the alternative one, denoted by $H_{1}$, that the vessel moves away from the nominal route once the AIS transponder has been shut down. At the end of the time interval $T$, the AIS device is switched back on and the vessel keeps on moving under the nominal condition, as shown in Fig. 2.

We assume that, during the time period when a lack of data occurs, the vessel had been moving according to a sequence of OU processes with unknown long-run mean velocities or, equivalently, a single OU process with long-run mean velocity that is a piecewise-constant function of time. In Fig. 2 it is shown the sequence of long-run mean velocities, which identify a $N$-section path, that we represent with the vector $\boldsymbol{v}_{1: N}=\left[\boldsymbol{v}_{1}^{\mathrm{T}} \ldots \boldsymbol{v}_{N}^{\mathrm{T}}\right]^{\mathrm{T}} \in \mathbb{R}^{2 N}$. Considering the set of time instants $t_{1}<\ldots<t_{n}<\ldots<t_{N}$, the period taken to cover the $n$-th section corresponds to the difference $\Delta_{n}=t_{n}-t_{n-1}$.

In other words, under $H_{1}$, the vessel velocity is modeled as a piecewise $O U$ model, i.e. an $\mathrm{OU}$ process with a long-run mean velocity parameter that is a piecewise-constant function of time. The time period $T$, during which the AIS is disabled, can be expressed as the sum of all the different time intervals $\Delta_{n}$, so that $T=\sum_{n=1}^{N} \Delta_{n}$.

The considered problem amounts determining, in absence of AIS data and without any other information during the time interval $T$, whether the vessel has been following the planned trajectory at the nominal velocity $\boldsymbol{v}_{0}$ or not, by means of a composite hypothesis testing formulation designed to identify changes in the velocity parameter.

\section{Detection STRATEGY}

\section{A. Ornstein-Uhlenbeck (OU) model}

Validated against a real-world commercial maritime traffic dataset, the OU model enables a more accurate representation of the target state in the long term, when ships are not maneuvering [5]. The OU process is distinguished from the conventional nearly-constant velocity (NCV) model mainly by a feedback loop, which ensures that the velocity of the target does not diverge with time, but is instead bounded around a desired value, i.e. the velocity of the process tends to drift over time towards its long-term mean. Let us indicate the fourdimensional target state at time $t \in \mathbb{R}_{0}^{+}$with

$$
\mathbf{s}(t)=[\mathbf{x}(t) \dot{\mathbf{x}}(t)]^{\mathrm{T}},
$$

where $\mathbf{x}(t)$ and $\dot{\mathbf{x}}(t)$ denote the target position and velocity, respectively, in a two-dimensional Cartesian reference system

$$
\mathbf{x}(t) \triangleq[x(t) y(t)], \dot{\mathbf{x}}(t) \triangleq[\dot{x}(t) \dot{y}(t)] .
$$

The target dynamics, in general, are modeled by a set of linear stochastic differential equations (SDEs) [12], and in [5] it is shown how the movement of real non-maneuvering vessels in the open sea can be represented by a mean-reverting stochastic process. Specifically, the velocity of the target is 
an OU process, and its position is an Integrated OU (IOU) process. Under this assumption, the SDE for the target motion model has the following form

$$
d \mathbf{s}(t)=\mathbf{A} \mathbf{s}(t) d t+\mathbf{G} \boldsymbol{v} d t+\mathbf{B} d \boldsymbol{\omega}(t),
$$

where $\boldsymbol{v}=\left[\begin{array}{ll}v_{x} & v_{y}\end{array}\right]^{\mathrm{T}}$ is the long-run process mean, and $\boldsymbol{\omega}(t)$ is a standard bi-dimensional Wiener process. The matrices $\mathbf{A}$, $\mathbf{B}$ and $\mathbf{G}$ are defined as:

$$
\mathbf{A}=\left[\begin{array}{cc}
\mathbf{0} & \mathbf{I} \\
\mathbf{0} & -\boldsymbol{\Theta}
\end{array}\right], \quad \mathbf{B}=\left[\begin{array}{c}
\mathbf{0} \\
\boldsymbol{\Sigma}
\end{array}\right], \quad \mathbf{G}=\left[\begin{array}{c}
\mathbf{0} \\
\boldsymbol{\Theta}
\end{array}\right],
$$

where $\mathbf{0}$ is a $2 \times 2$ null matrix, $\boldsymbol{\Sigma}$ is a $2 \times 2$ matrix defining the noise process and $\Theta$ is a $2 \times 2$ matrix quantifying the meanreversion effect, meaning the rate at which the target will tend back to the desired velocity after a perturbation; its diagonal terms refer to the $x$ and $y$ components, while the off-diagonals quantify the coupling effect.

Unless otherwise stated, we will use hereafter subscripted indices to denote time dependency, i.e. $\mathbf{x}_{n}=\mathbf{x}\left(t_{n}\right), \dot{\mathbf{x}}_{n}=$ $\dot{\mathbf{x}}\left(t_{n}\right)$ and $\mathbf{s}_{n}=\mathbf{s}\left(t_{n}\right)$ by definition. We also assume that $\Theta$ is diagonalizable and has positive eigenvalues, so that an affine transformation can be found that projects the matrix $\boldsymbol{\Theta}$ onto another space, i.e. $\boldsymbol{\Theta}=\mathbf{R} \boldsymbol{\Gamma} \mathbf{R}^{-1}$, where $\mathbf{R}$ is the matrix whose columns contain the eigenvectors of $\boldsymbol{\Theta}$ and $\boldsymbol{\Gamma}$ is a diagonal matrix whose elements are the corresponding eigenvalues. This idea is expanded further in [5], where the general solution to the coupled problem is provided. For the sake of simplicity and without loss of generality we assume that $\mathbf{R}=\mathbf{I}$, so that $\boldsymbol{\Theta}=\boldsymbol{\Gamma}=\operatorname{diag}(\boldsymbol{\gamma})$, with $\gamma=\left[\gamma_{x}, \gamma_{y}\right]^{\mathrm{T}}$. In other words, as described in [5], there are three parameters for each coordinate: the long-run mean velocity $\boldsymbol{v}$, the reversion rate $\gamma$ and the process noise $\sigma$.

The model can be easily extended to the case of waypoint navigation [7], that is relevant to our application, being the navigation mode of substantially all the commercial maritime traffic. Under this case, we can assume that the long-run mean velocity of the target is a piecewise-constant function of the time that takes values from a sequence $\boldsymbol{v}_{1}, \ldots, \boldsymbol{v}_{N}$. Along the navigational legs, the long-run mean velocity of the target follows, by all means, an OU process; conversely, the maneuver is represented by a change of the long-run mean velocity parameter. Under these assumptions, the target state at time $t_{i}$, given the target state at the previous $i-1$ times can be written, in matrix form as

$$
\mathbf{s}_{i}=\boldsymbol{\Phi}\left(t_{i}-t_{i-1}, \gamma\right) \mathbf{s}_{i-1}+\boldsymbol{\Psi}\left(t_{i}-t_{i-1}, \gamma\right) \boldsymbol{v}_{i}+\boldsymbol{\omega}_{i}
$$

where $\boldsymbol{v}_{i}$ is the long-run mean velocity in the time interval $\Delta_{i}=\left[t_{i-1}, t_{i}\right]$ and $\boldsymbol{\omega}_{i}=\boldsymbol{\omega}\left(\Delta_{i}\right)$ is a zero-mean Gaussian random variable with covariance $\mathbf{C}\left(\Delta_{i}\right)$ defined in [5]. The state transition matrix and the control input function, $\boldsymbol{\Phi}(\Delta, \gamma)$ and $\Psi(\Delta, \gamma)$, respectively, are defined as

$$
\begin{gathered}
\boldsymbol{\Phi}\left(\Delta_{i}, \gamma\right)=\left[\begin{array}{cc}
\mathbf{I} & \left(\mathbf{I}-e^{-\boldsymbol{\Gamma} \Delta_{i}}\right) \boldsymbol{\Gamma}^{-1} \\
\mathbf{0} & e^{-\boldsymbol{\Gamma} \Delta_{i}}
\end{array}\right], \\
\boldsymbol{\Psi}\left(\Delta_{i}, \gamma\right)=\left[\begin{array}{c}
\Delta_{i} \mathbf{I}-\left(\mathbf{I}-e^{-\boldsymbol{\Gamma} \Delta_{i}}\right) \boldsymbol{\Gamma}^{-1} \\
\mathbf{I}-e^{-\boldsymbol{\Gamma} \Delta_{i}}
\end{array}\right],
\end{gathered}
$$

whose derivations are provided in [5].

The target state at time $t_{N}$, given the target states at the previous $N-1$ times can be expressed recursively as (omitting $\gamma$ for clarity)

$$
\begin{aligned}
\mathbf{s}_{N}= & \boldsymbol{\Phi}\left(\Delta_{N}\right) \mathbf{s}_{N-1}+\boldsymbol{\Psi}\left(\Delta_{N}\right) \boldsymbol{v}_{N}+\boldsymbol{\omega}_{N} \\
= & \boldsymbol{\Phi}\left(\Delta_{N}\right)\left[\boldsymbol{\Phi}\left(\Delta_{N-1}\right) \mathbf{s}_{N-2}+\boldsymbol{\Psi}\left(\Delta_{N-1}\right) \boldsymbol{v}_{N-1}+\boldsymbol{\omega}_{N-1}\right] \\
& +\boldsymbol{\Psi}\left(\Delta_{N}\right) \boldsymbol{v}_{N}+\boldsymbol{\omega}_{N}=\ldots \\
= & \boldsymbol{\Phi}(T) \mathbf{s}_{0}+\boldsymbol{\Psi}\left(\Delta_{N}\right) \boldsymbol{v}_{N}+\boldsymbol{\omega}_{N}+ \\
& +\sum_{n=1}^{N-1}\left[\prod_{i=n+1}^{N} \boldsymbol{\Phi}\left(\Delta_{i}\right)\right]\left[\boldsymbol{\Psi}\left(\Delta_{n}\right) \boldsymbol{v}_{n}+\boldsymbol{\omega}_{n}\right]
\end{aligned}
$$

where we exploited the property of the state transition matrix by which $\boldsymbol{\Phi}\left(\Delta_{1}\right) \boldsymbol{\Phi}\left(\Delta_{2}\right) \ldots \boldsymbol{\Phi}\left(\Delta_{n}\right)=\boldsymbol{\Phi}\left(\Delta_{1}+\Delta_{2}+\ldots+\Delta_{n}\right)$, $\forall n=1, \ldots, N$, that can be derived by inspection from (6).

\section{B. Statistical representation of data based on OU model}

The two measurements, denoted with $\mathbf{z}$ and $\mathbf{z}_{0}$, are available, respectively at time $T$ and time $t_{0}$

$$
\mathbf{z}=\mathbf{s}(T)+\mathbf{n}, \quad \mathbf{z}_{0}=\mathbf{s}\left(t_{0}\right)+\mathbf{n}_{0},
$$

where $\mathbf{n}$ and $\mathbf{n}_{0}$ are independent zero-mean Gaussian noises with covariance matrices $\mathbf{C}_{\mathbf{n}}$ and $\mathbf{C}_{\mathbf{n}_{0}}$, respectively. Clearly, the measurement noise is independent from the OU process noise. Given the linearity of (9) and the fact that $\mathbf{z}$ is Gaussian, we can use the following vector of data to avoid the dependence on $\mathbf{s}\left(t_{0}\right)$ in $\mathbf{z}$

$$
\begin{aligned}
\mathbf{y}= & \mathbf{z}-\boldsymbol{\Phi}(T) \mathbf{z}_{0} \\
= & \boldsymbol{\Psi}\left(\Delta_{N}\right) \boldsymbol{v}_{N}+\boldsymbol{\omega}_{N} \\
& +\sum_{n=1}^{N-1}\left[\prod_{i=n+1}^{N} \boldsymbol{\Phi}\left(\Delta_{i}\right)\right]\left[\boldsymbol{\Psi}\left(\Delta_{n}\right) \boldsymbol{v}_{n}+\boldsymbol{\omega}_{n}\right] \\
& +\mathbf{n}-\boldsymbol{\Phi}(T) \mathbf{n}_{0} .
\end{aligned}
$$

The terms $\boldsymbol{\omega}_{n}=\boldsymbol{\omega}\left(\Delta_{n}\right)$ are independent zero-mean Gaussian random variables with covariance $\mathbf{C}\left(\Delta_{n}\right)$ [5]. As shown in [4], it amounts to compute

$\boldsymbol{\omega}(T) \triangleq \sum_{n=1}^{N-1}\left[\prod_{i=n+1}^{N} \boldsymbol{\Phi}\left(\Delta_{i}\right)\right] \boldsymbol{\omega}\left(\Delta_{n}\right)+\boldsymbol{\omega}\left(\Delta_{N}\right) \sim \mathcal{N}(\mathbf{0}, \mathbf{C}(T))$

so that $\mathbf{y}$ can be recast as follows

$$
\mathbf{y}=\boldsymbol{\theta}+\boldsymbol{\omega}(T)+\mathbf{n}-\boldsymbol{\Phi}(T) \mathbf{n}_{0} \sim \mathcal{N}\left(\boldsymbol{\theta}, \mathbf{C}_{\mathbf{y}}\right),
$$

where the expression of the mean term $\boldsymbol{\theta}$ is given by

$$
\boldsymbol{\theta} \triangleq \sum_{n=1}^{N-1}\left[\prod_{i=n+1}^{N} \boldsymbol{\Phi}\left(\Delta_{i}\right)\right] \boldsymbol{\Psi}\left(\Delta_{n}\right) \boldsymbol{v}_{n}+\boldsymbol{\Psi}\left(\Delta_{N}\right) \boldsymbol{v}_{N}
$$

while, the covariance matrix is given by

$$
\mathbf{C}_{\mathbf{y}} \triangleq \mathbf{C}(T)+\mathbf{C}_{\mathbf{n}}+\boldsymbol{\Phi}(T) \mathbf{C}_{\mathbf{n}_{0}} \boldsymbol{\Phi}(T)^{\mathrm{T}},
$$

assuming the independence of the terms in (11). 


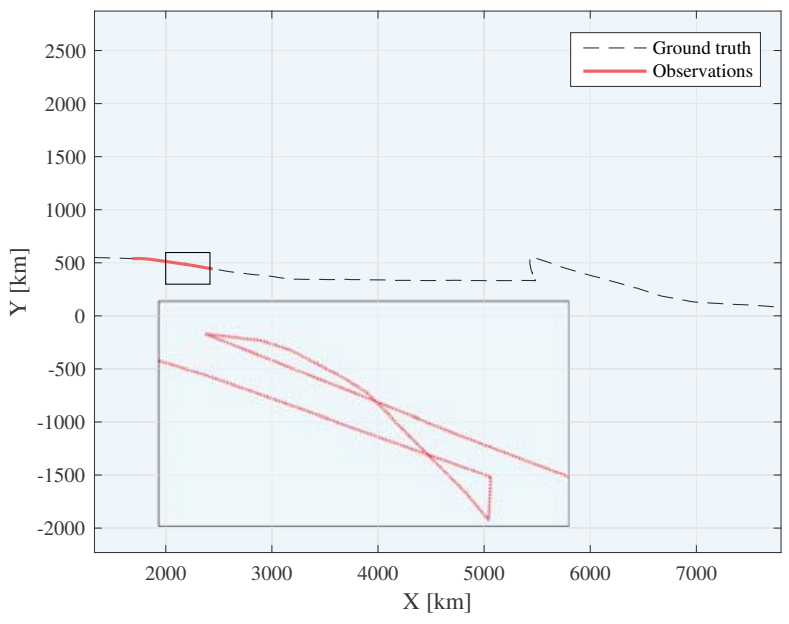

Fig. 3. Complete AIS track ( $1^{\text {st }}$ region).

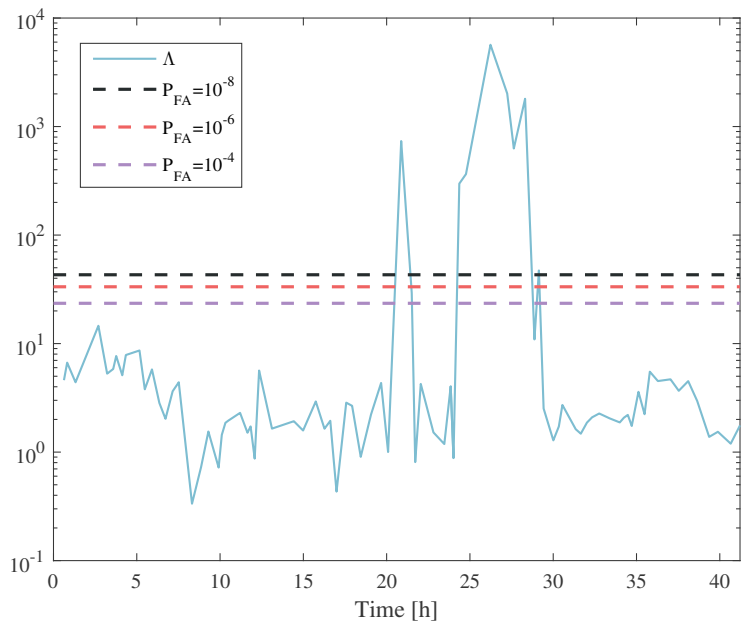

Fig. 5. Test statistic (16) related to the complete AIS track ( $1^{\text {st }}$ region).

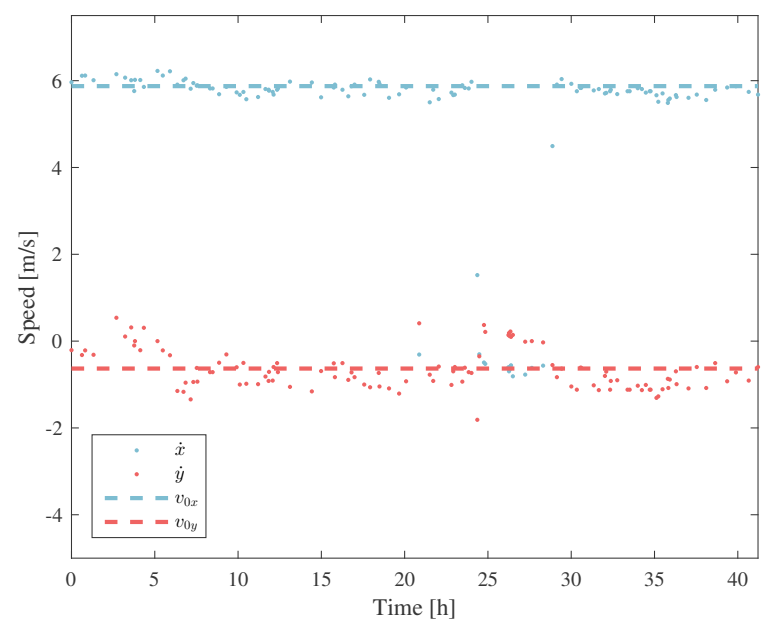

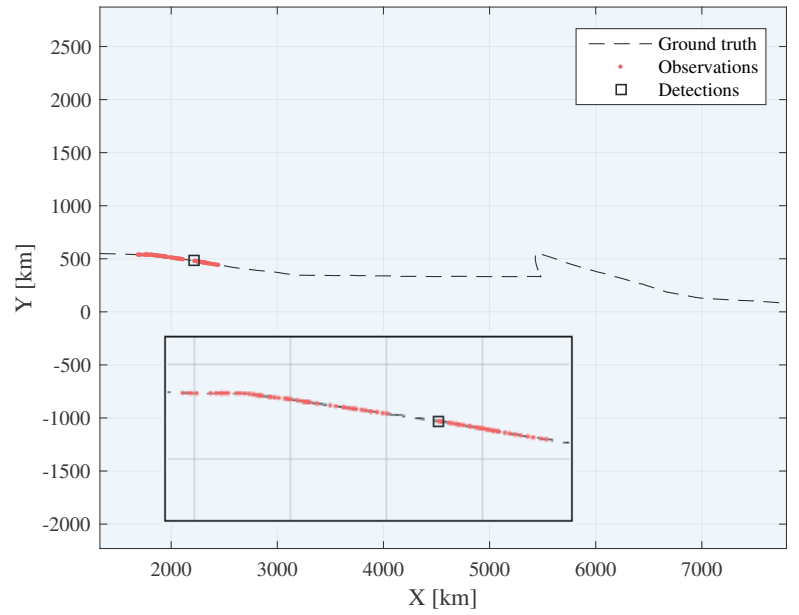

Fig. 4. Simulated gap in the AIS track ( $1^{\text {st }}$ region).

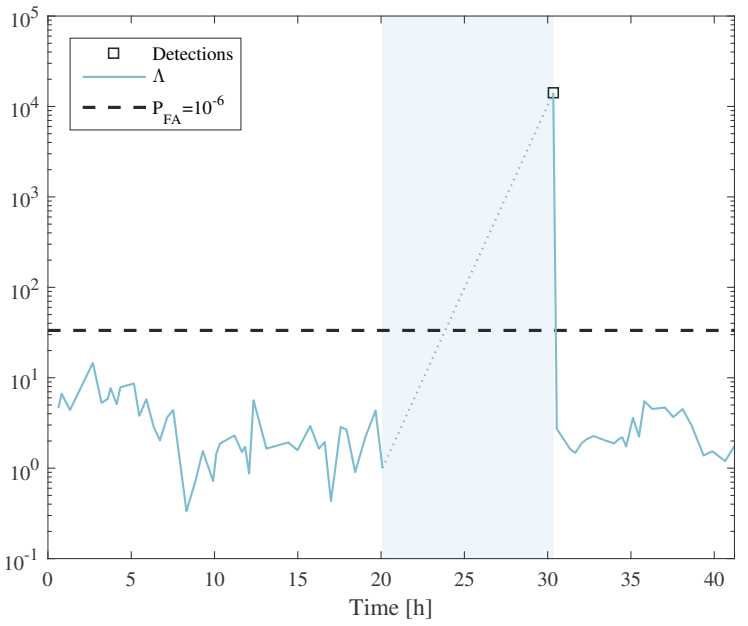

Fig. 6. Test statistic (16) related to the incomplete AIS track (1 ${ }^{\text {st }}$ region).

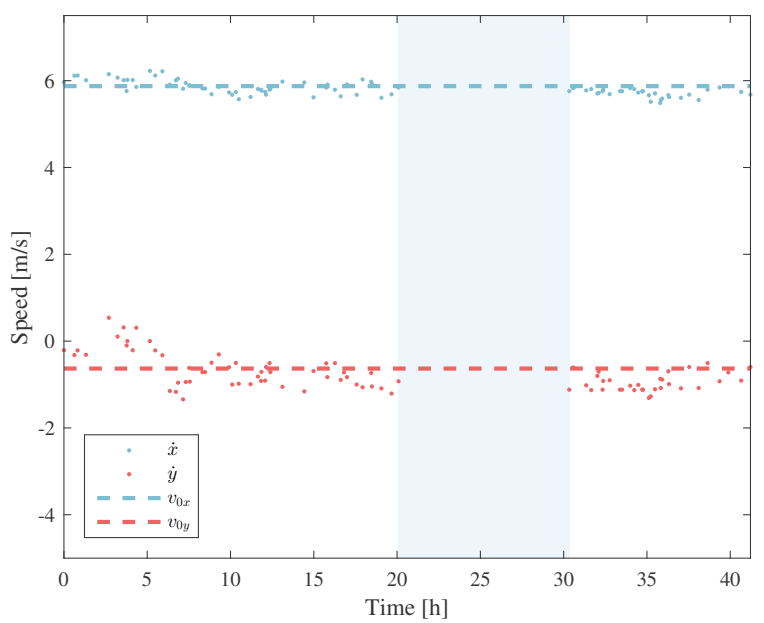

Fig. 7. Velocity components related to the complete AIS track ( $1^{\text {st }}$ region). Fig. 8. Velocity components related to the incomplete AIS track ( ${ }^{\text {st }}$ region). 


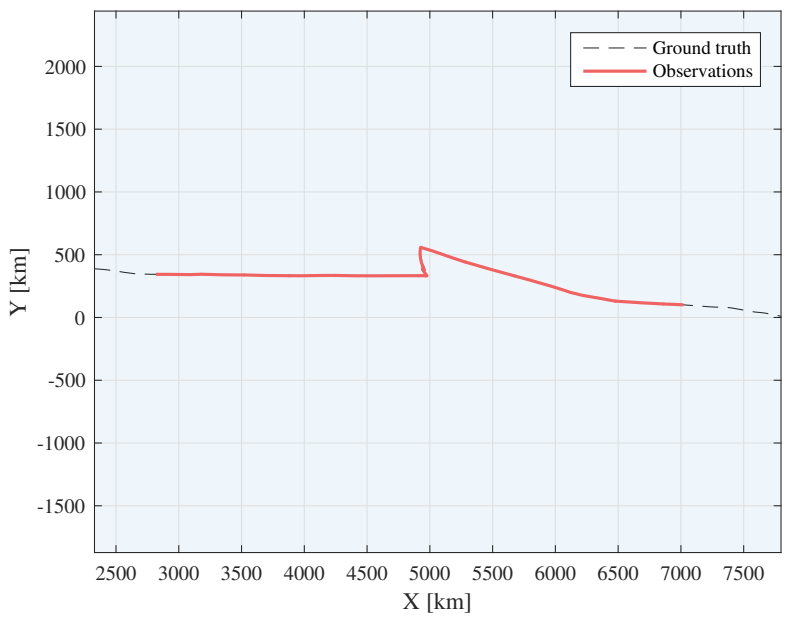

Fig. 9. Complete AIS track ( $2^{\text {nd }}$ region).

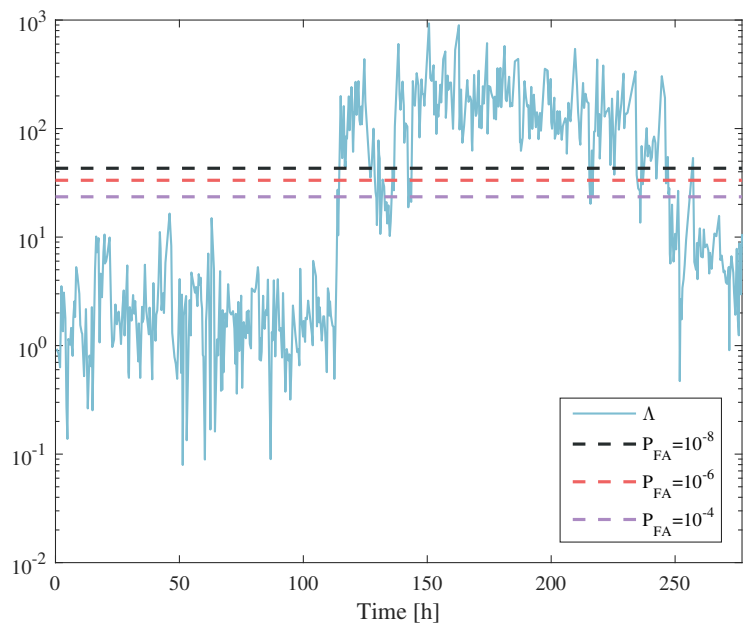

Fig. 11. Test statistic (16) related to the complete AIS track ( $2^{\text {nd }}$ region).

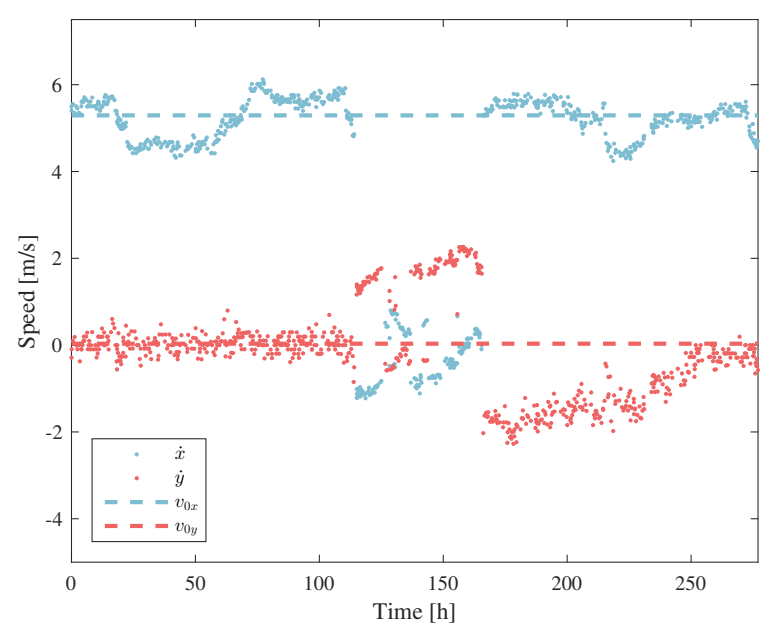

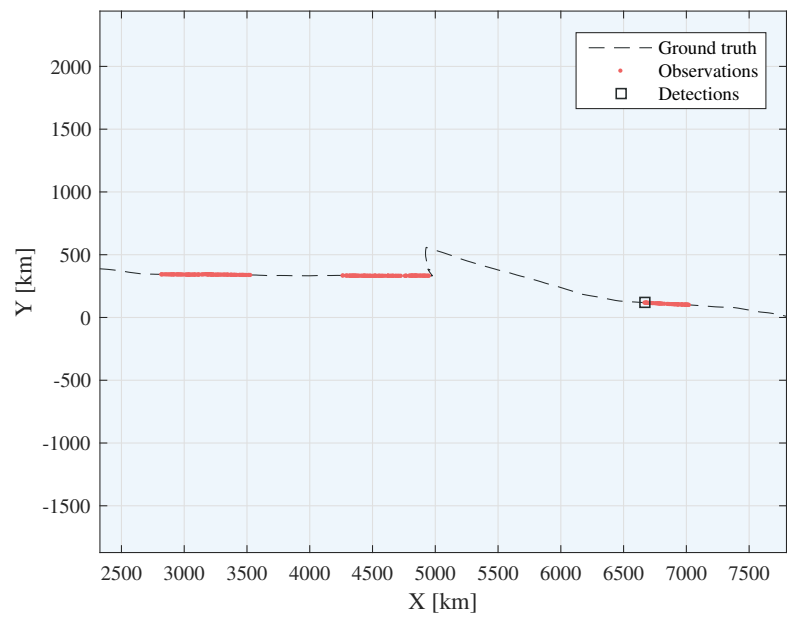

Fig. 10. Simulated gaps in the AIS track ( $2^{\text {nd }}$ region).

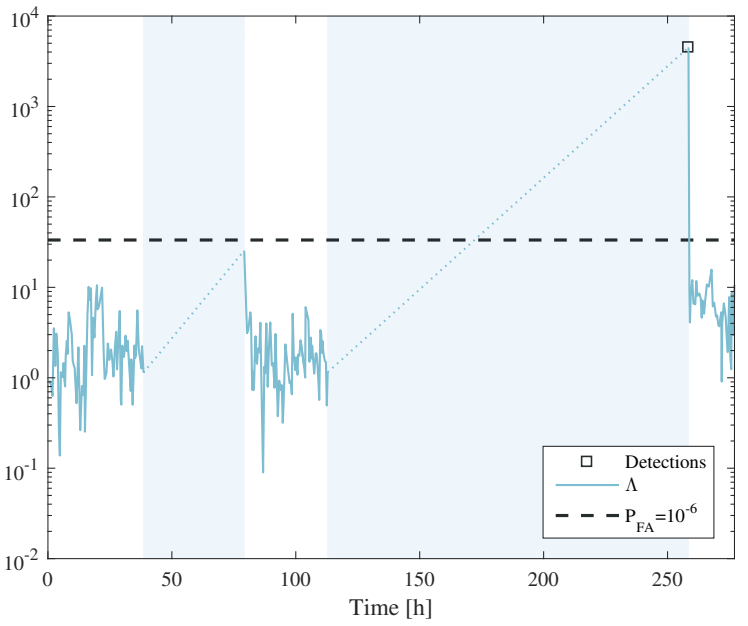

Fig. 12. Test statistic (16) related to the incomplete AIS track ( $2^{\text {nd }}$ region).

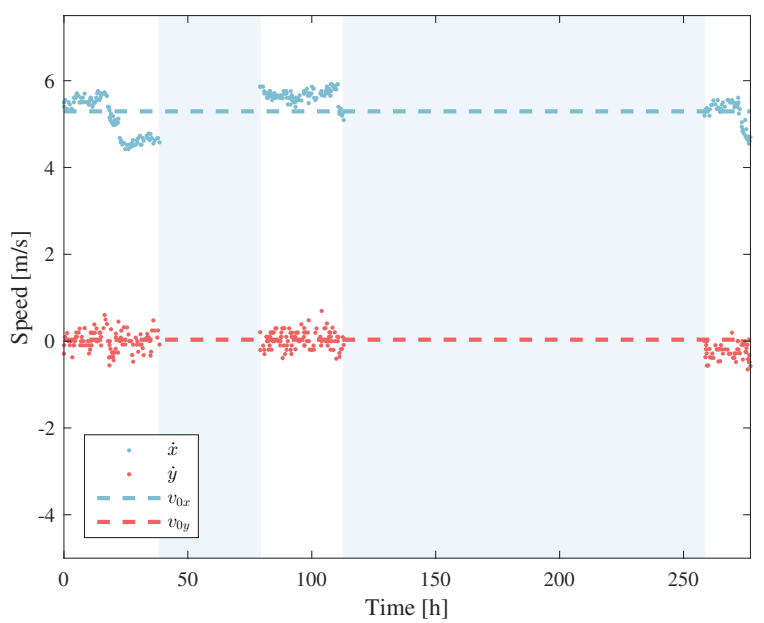

Fig. 13. Velocity components related to the complete AIS track ( $2^{\text {nd }}$ region). Fig. 14 . Velocity components related to the incomplete AIS track (2 ${ }^{\text {nd }}$ region). 


\section{Detection strategy performance}

The anomaly detection problem can be traced back to the following Gaussian composite hypothesis testing problem:

$$
\left\{\begin{array}{l}
H_{0}: \mathbf{y} \sim \mathcal{N}\left(\boldsymbol{\theta}_{0}, \mathbf{C}_{\mathbf{y}}\right) \\
H_{1}: \mathbf{y} \sim \mathcal{N}\left(\boldsymbol{\theta}, \mathbf{C}_{\mathbf{y}}\right)
\end{array}\right.
$$

where the mean term $\boldsymbol{\theta}$ under $H_{1}$ is given in (12), while, under the null hypothesis $H_{0}$, it is possible to show that $\boldsymbol{\theta}_{0} \triangleq$ $\boldsymbol{\Psi}(T) \boldsymbol{v}_{0}$ by exploiting the following equality

$$
\sum_{n=1}^{N-1}\left[\prod_{i=n+1}^{N} \boldsymbol{\Phi}\left(\Delta_{i}\right)\right] \boldsymbol{\Psi}\left(\Delta_{n}\right)+\boldsymbol{\Psi}\left(\Delta_{N}\right)=\boldsymbol{\Psi}(T) .
$$

The anomaly detection strategy investigated is the Generalized Likelihood Ratio Test (G-LRT) [13]-[15] and, assuming the matrix $\mathbf{C}_{\mathbf{y}}$ is invertible, it is derived as follows,

$$
\left(\widehat{\boldsymbol{\theta}}-\boldsymbol{\theta}_{0}\right)^{\mathrm{T}} \mathbf{C}_{\mathbf{y}}^{-1}\left(\widehat{\boldsymbol{\theta}}-\boldsymbol{\theta}_{0}\right) \stackrel{H_{1}}{\underset{H_{0}}{\gtrless}} \tau,
$$

where $\widehat{\boldsymbol{\theta}}=\mathbf{y}$ represents the ML estimate for the parameter $\boldsymbol{\theta}$.

The test statistics under the two hypotheses $H_{0}$ and $H_{1}$ are characterized, respectively, by a central and a non-central Chisquared distributions, both with $d=4$ degrees of freedom, corresponding to the size of the known parameter $\boldsymbol{\theta}_{0}$. The detection performance is therefore given by

$$
\begin{gathered}
\mathrm{P}_{\mathrm{FA}}=Q_{\chi_{d}^{2}}(\tau), \\
\mathrm{P}_{\mathrm{D}}=Q_{\chi_{d}^{\prime 2}(\lambda)}(\tau),
\end{gathered}
$$

where $Q_{\chi_{d}^{2}}$ and $Q_{\chi_{d}^{\prime 2}(\lambda)}$ are the right tail probabilities of the central and non-central Chi-squared distributions, respectively, with the following non-centrality parameter

$$
\lambda=\left(\boldsymbol{\theta}-\boldsymbol{\theta}_{0}\right)^{\mathrm{T}} \mathbf{C}_{\mathbf{y}}^{-1}\left(\boldsymbol{\theta}-\boldsymbol{\theta}_{0}\right) .
$$

\section{REAL SCENARIO ANALYSIS}

The strategy proposed in this work has been applied to real AIS data corresponding to the track shown in Fig. 1, where two regions of anomalous behavior can be identified by simple visual inspection.

For the application of the detection strategy, the OU parameters have been estimated in the path section immediately preceding the one where the deviation from the nominal conditions actually happens. Specifically, the estimated values of the process noise $\boldsymbol{\sigma}$, the long-run mean velocity $\boldsymbol{v}_{0}$, and the reversion rate $\gamma$ are reported in Table I.

\section{A. First region}

The anomalous behavior in the first region is not very apparent and short. According to the AIS track, the vessel seems to shut down the engines and drift for about 14 hours, then it goes back to the planned route as it can be seen in the close-up of Fig. 3. Such an anomaly is reflected on the velocity components shown in Fig. 7, but it is more visible in Fig. 5 where, during the deviation time, the decision statistic (16) grows by several orders of magnitude with respect
TABLE I

OU PARAMETERS ESTIMATES

\begin{tabular}{|c|c|c|c|}
\cline { 3 - 4 } \multicolumn{2}{c|}{} & First region & Second region \\
\hline \multirow{2}{*}{$\gamma$} & $\gamma_{x}$ & $5.89 \times 10^{-3}$ & $2.30 \times 10^{-4}$ \\
\cline { 2 - 4 } & $\gamma_{y}$ & $8.49 \times 10^{-4}$ & $4.19 \times 10^{-3}$ \\
\hline \multirow{2}{*}{$\boldsymbol{\sigma}$} & $\sigma_{x}$ & $2.83 \times 10^{-2}$ & $1.13 \times 10^{-2}$ \\
\cline { 2 - 4 } & $\sigma_{y}$ & $1.84 \times 10^{-2}$ & $2.23 \times 10^{-2}$ \\
\hline \multirow{2}{*}{$\boldsymbol{v}_{0}$} & $v_{0_{x}}$ & 5.8743 & 5.2931 \\
\cline { 2 - 4 } & $v_{0_{y}}$ & -0.6320 & 0.0331 \\
\hline
\end{tabular}

to nominal values, exceeding all the values of the threshold (i.e. for different values of the false alarm probability: $\mathrm{P}_{\mathrm{FA}} \in$ $\left.\left\{10^{-4}, 10^{-6}, 10^{-8}\right\}\right)$.

A gap has been simulated in the AIS track, corresponding to the anomaly time frame, as depicted in Fig. 4, in order to test the proposed detection strategy. The detector (16) correctly reveals the deviation from the nominal condition, using a threshold selected with $\mathrm{P}_{\mathrm{FA}}=10^{-6}$, as shown in Fig. 6 .

\section{B. Second region}

The AIS track of the ship in the second observation window is shown in Fig. 9 and it does indeed reveal a deviation from the normal route during a time frame of about 5 days; Fig. 11 displays the test statistic (16), which exceeds the threshold (plotted for different values of the false alarm probability in the same range considered for the first region analysis) corresponding to the deviation from the nominal condition. In particular, the deviation from the nominal velocity is visible in Fig. 13 where the velocity components show an apparent change in that specific time frame.

As done for the first region, the detection strategy is tested with simulated gaps in AIS data, as shown in Fig. 10, with the corresponding velocity gaps shown in Fig 14. The first gap occurs in a section of the trajectory where there is no deviation from the nominal conditions, while the second one occurs where the deviation actually happens. From the application of the detector (16) with $\mathrm{P}_{\mathrm{FA}}=10^{-6}$, the deviation can be properly detected while no detection is correctly declared in the first gap, as shown in Fig. 12.

\section{CONCLUSION}

A maritime anomaly detection problem has been studied assuming an Ornstein-Uhlenbeck (OU) mean-reverting stochastic motion model for the vessel dynamics. The aim was to reveal a possible deviation of the vessel under consideration from its nominal conditions, during an AIS device disablement, relying on a hypothesis testing procedure based on the generalized likelihood ratio test that builds on the changes in the OU process long-term velocity parameter.

A closed-form expression has been provided for the detector, the false alarm probability, and the anomaly detection 
probability, expressed in terms of a central and a non-central chi-squared distributions, respectively.

Finally, the proposed detection strategy has been applied to two different sections of a real trajectory of a ship that performs deviations from her nominal route; within the trajectory, data gaps have been simulated in correspondence of and outside the deviation. The two hypothesized anomalies appear to be fairly difficult to be identified by the simple visual inspection of a human operator, who might not be able to reveal such small-scale deviations within a big picture. Therefore, the anomaly detection algorithm appears to be useful also in practical situations, as it could be applied automatically and simultaneously to several trajectories in order to reveal possible deviations.

\section{ACKNOWLEDGMENT}

This work was supported by the NATO Allied Command Transformation (ACT) via the project Data Knowledge Operational Effectiveness (DKOE) at the NATO Science and Technology Organization (STO) Centre for Maritime Research and Experimentation (CMRE). The authors thankfully acknowledge MarineTraffic and Prof. Dimitris Zissis from the University of the Aegean for providing the real-world AIS data set used for the experimental analysis and for the valuable domain expert insights.

\section{REFERENCES}

[1] M. Jakob, O. Vaňek, S. Urban, P. Benda, and M. Pěchouček, "Adversarial modeling and reasoning in the maritime domain," Agent Technology Center, Department of Cybernetics, FEE Czech Technical University in Prague, Tech. Rep., December 2009.

[2] R. O. Lane, D. A. Nevell, S. D. Hayward, and T. W. Beaney, "Maritime anomaly detection and threat assessment," in 2010 13th International Conference on Information Fusion, July 2010, pp. 1-8.

[3] J. J. Alava, M. J. Barragán-Paladines, J. Denkinger, L. Muñoz-Abril, P. Jiménez, F. Paladines, and et al., "Massive Chinese fleet jeopardizes threatened shark species around the Galápagos marine reserve and waters off Ecuador: Implications for national and international fisheries policy," International Journal of Fisheries Sci Res., 2017.

[4] E. d'Afflisio, P. Braca, L. M. Millefiori, and P. Willett, "Detecting stealth deviations from standard routes using the Ornstein-Uhlenbeck process," submitted.

[5] L. M. Millefiori, P. Braca, K. Bryan, and P. Willett, "Modeling vessel kinematics using a stochastic mean-reverting process for long-term prediction," IEEE Transactions on Aerospace and Electronic Systems, vol. 52, no. 5, pp. 2313-2330, October 2016.

[6] L. M. Millefiori, P. Braca, and P. Willett, "Consistent estimation of randomly sampled Ornstein-Uhlenbeck process long-run mean for longterm target state prediction." IEEE Signal Processing Letters, vol. 23, no. 11, pp. 1562 - 1566, November 2016.

[7] P. Coscia, P. Braca, L. M. Millefiori, F. Palmieri, and P. Willett, "Maritime traffic representation based on sea-lanes graph construction criteria using multiple Ornstein-Uhlenbeck processes," IEEE Transactions on Aerospace and Electronic Systems, to be published, 2018.

[8] L. M. Millefiori, P. Braca, and G. Arcieri, "Scalable distributed change detection and its application to maritime traffic," in 2017 IEEE International Conference on Big Data (Big Data), Dec 2017, pp. 1650-1657.

[9] G. Vivone, L. M. Millefiori, P. Braca, and P. Willett, "Model performance assessment for long-term vessel prediction using HFSW radar data," in 2017 IEEE Radar Conference (RadarConf), May 2017, pp. 0243-0247.

[10] L. M. Millefiori, P. Braca, K. Bryan, and P. Willett, "Long-term vessel kinematics prediction exploiting mean-reverting processes," in 2016 19th International Conference on Information Fusion (FUSION), July 2016, pp. 232-239.
[11] G. Vivone, L. M. Millefiori, P. Braca, and P. Willett, "Performance assessment of vessel dynamic models for long-term prediction using heterogeneous data," IEEE Transactions on Geoscience and Remote Sensing, vol. 55, no. 11, pp. 6533-6546, Nov 2017.

[12] X. R. Li and V. P. Jilkov, "Survey of maneuvering target tracking. Part I. Dynamic models," IEEE Transactions on Aerospace and Electronic Systems, vol. 39, no. 4, pp. 1333-1364, Oct 2003.

[13] S. M. Kay, Fundamentals of Statistical Signal Processing, Volume II: Detection Theory. Prentice Hall PTR, 1998.

[14] A. Thorpe and L. Scharf, "Data adaptive rank-shaping methods for solving least squares problems," IEEE Transactions on Signal Processing, vol. 43, no. 7, pp. 1591-1601, july 1995 .

[15] B. Porat and B. Friedlander, "Performance analysis of a class of transient detection algorithms-a unified framework," IEEE Transactions on Signal Processing, vol. 40, no. 10, pp. 2536-2546, Oct 1992. 